\title{
Nanoclusters of crystallographically aligned nanoparticles for magnetic thermotherapy: aqueous ferrofluid, agarose phantom and ex vivo melanoma tumour assessment.
}

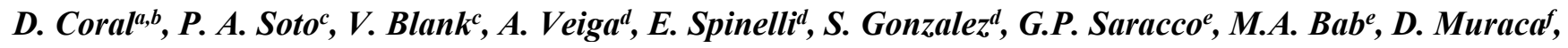 \\ P.C.Setton-Avruj', A. Roigg, L.Roguin', M. B. Fernández van Raap ${ }^{a}{ }^{\dagger}$
}

\begin{abstract}
Magnetic hyperthermia is an oncologic therapy where magnetic nanostructures, under a radiofrequency field, act as heat transducers increasing tumour temperature and killing cancerous cells. Nanostructure heating efficiency depends both on the field conditions and on the nanostructure properties and mobility inside the tumour. Such nanostructures are often incorrectly bench-marketed in colloidal state and using field settings far off from the recommended therapeutic values. Here, we prepared nanoclusters composed of iron oxide magnetite nanoparticles crystallographically aligned and their specific absorption rate (SAR) values were calorimetrically determined in physiological fluids, agarose-gel-phantoms and ex vivo tumours extracted from mice challenged with B16-F0 melanoma cells. A portable, multipurpose applicator using medical field settings; $100 \mathrm{kHz}$ and $9.3 \mathrm{kA} \mathrm{m}^{-1}$, was developed and the results were fully analysed in terms of nanoclusters structural and magnetic properties. A careful appraisal of the nanoclusters heating capacity in the three milieus clearly indicate that the SAR values of fluid suspensions or agarose-gel-phantoms are not adequate to predict real tissue temperature rise or the dosage needed to heat a tumour. Our results show that besides nanostructure mobility, perfusion and local thermoregulation, nanostructures distribution inside the tumour plays a key role in the effective heating. A suppression of magnetic material effective heating efficiency appears in tumour tissue. In fact, dosage had to be much increased, from the SAR values predicted from fluid or agarose to achieve the desired temperature increase. These results represent an important contribution towards the design of more efficient nanostructures and towards clinical translation of hyperthermia.
\end{abstract}

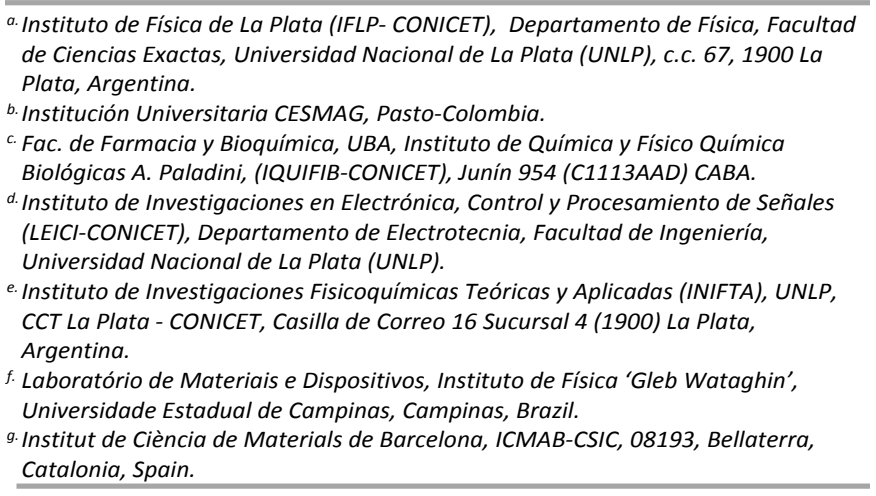

† Corresponding author: raap@fisica.unlp.edu.ar, phone: +54 221 4246062, Fax +54 2214236335

Electronic Supplementary Information (ESI) available: Procedures for M vs. H curves for fitting and SAR values semi-empirical calculation. Field inductor design discussion. Fig. S1: TEM histograms. Fig. S2-S7: HRTEM NCs images and FFT. Fig. S8: Magnetization curves at $300 \mathrm{~K}$. Fig. S9: heating curves of aqueous nanoclusters suspensions. Fig S10. Cryo-TEM images of B16-F0 cells incubated with NCs. See DOI: $10.1039 / x 0 x x 00000 x$

\section{Introduction}

Colloidal dispersions of magnetic nanoparticles (MNPs) in physiological fluids have shown great potential as heat mediators for hyperthermia oncologic therapy ${ }^{1}$. Briefly, magnetic hyperthermia $(\mathrm{MH})$ is the modality among thermotherapies that use MNPs and an alternating magnetic field (AMF) in the radiofrequency range to increase solid tumours temperature. The MNPs absorbs energy from the field to release it to its environment through Néel and Brown relaxation mechanisms ${ }^{2}$.

$\mathrm{MH}$ relies on two facts: large doses of intratumourally-injected nanoparticles can generate enough heat to inflict damage to cancer cells and that the overall blood flow tends to be significantly lower in certain poorly vascularized tumour areas with respect to wellvascularized normal tissues ${ }^{3}$, ensuring tumour selectivity by minimizing heating of adjacent healthy tissue. Tumour cancer cells apoptosis is induced when tissue temperature reaches therapeutic values $\left(43-45^{\circ} \mathrm{C}\right)^{4}$ whilst cell/tumour ablation occurs above $45{ }^{\circ} \mathrm{C}$. Tissue temperature elevation can also be used to improve cancer cells sensitivity to radiation and/or to anticancer drugs. $\mathrm{MH}$ recently received EU-wide regulatory approval as medical devices for the 
treatment of brain tumours. Previous clinical trials of $\mathrm{MH}$ combined with radiotherapy 5,6 indicated survival benefit of 7.2 and 8.6 months for first tumour recurrence and primary tumour, respectively. Those results reinforce the idea that improvement in the detailed understanding of this procedure is still needed.

The most widely used nanomaterial in hyperthermia is iron oxide (magnetite and maghemite nanoparticles) due to its low cytotoxicity ${ }^{7}$, large biocompatibility and approval by the FDA and EMA. Over the last years, efforts on MNPs-mediated hyperthermia have been directed to reach specific absorption rate (SAR) values close to $1 \mathrm{~kW} \mathrm{~g}^{-1}$. As SAR increases with increasing field frequency $f$ and amplitude $H_{0}$, a very careful attention must be paid to these values. Indeed, a too strong field may generate Eddy currents and cause non-specific heating and therefore damaging healthy tissues and overheating of cancer tissues. In spite of this, many research groups still characterize their newly produced colloids at $f$ and $H_{0}$ values highly exceeding the recommended threshold value for major discomfort ${ }^{8,9}$, given by $H_{0} f<5 \times 10^{8} \mathrm{~A} \mathrm{~m}^{-1} \mathrm{~s}^{-1}$, and even over the more conservative value ${ }^{10}$ of $H_{0} f<50 \times 10^{8} \mathrm{~A} \mathrm{~m}^{-1} \mathrm{~s}^{-1}$. Besides Atkinson ${ }^{9}$ clinical tolerance test, performed in healthy volunteers, the first clinical application of interstitial hyperthermia, using MNPs in locally recurrent prostate cancer, indicated that pre-treatment planning had to be altered because the patients experienced discomfort when the field strength was increased above $5 \mathrm{kA} \mathrm{m}^{-1}$ at a frequency of 100 $\mathrm{kHz}^{11}$, clinically establishing a particular biologically safe range. Note that treatments in human patients are carried out at specially designed applicator of variable field amplitude lower than $18 \mathrm{kA} \mathrm{m}^{-1}$ and fixed frequency of $100 \mathrm{kHz}^{12}$.

In addition, MNPs characterization at high fields lead also to the problem that heating potential could later be insufficient for applications under field clinical conditions. Indeed, the higher the SAR, the lower the dosage needed to achieve a therapeutic temperature, which is crucial because too high dosages may induce significant toxicity and clearance issues ${ }^{13}$.

The various strategies tested to enhance the heating power, mainly proceed by varying MNPs size, shape, composition and more recently their structuration. Below, we briefly review previous results, we stress the fact that nanostructure optimization has been systematically carried out at field values far away from clinical settings and with MNPs dispersed in liquids, a model very far from a real tumour scenario.

Regarding size, crystalline spherical magnetite nanoparticles with core sizes between 20 and $30 \mathrm{~nm}$, in the transition zone between single magnetic domain and multidomain ${ }^{14}$ were proposed as well suited for $\mathrm{MH}$ with SAR value about $300 \mathrm{~W} \mathrm{~g}^{-1}$ for $22 \mathrm{~nm}$ at 77 $\mathrm{kHz}$ and $40 \mathrm{kA} \mathrm{m}^{-1}$ i.e. at a field setting corresponding to $H_{0} f$ value equal to 6.2 times the threshold value (TV) of $5 \times 10^{8} \mathrm{~A} \mathrm{~m}^{-1} \mathrm{~s}^{-1}$. Cubic shape particles also display large SAR values due to shape anisotropy contribution ${ }^{15,16}$. Guardia et al ${ }^{15}$ reported $509 \mathrm{~W} \mathrm{~g}^{-1}$ at $320 \mathrm{kHz}$ and 15 $\mathrm{kAm}^{-1}$ (9.6 times the TV) for a $19 \mathrm{~nm}$ cubic nanoparticle and MartinezBoubeta et al ${ }^{16}$ reported $150 \mathrm{~W} \mathrm{~g}^{-1}$ at $765 \mathrm{kHz}$ and $12 \mathrm{kA} \mathrm{m}^{-1}, 18$ times the TV, for $20 \mathrm{~nm}$ cubic particle. The nanostructuration of single-core magnetite to multi-core structure resulted in a SAR increase from $150 \mathrm{~W} \mathrm{~g}^{-1}$ to $1604 \mathrm{~W} \mathrm{~g}^{-1}$ under field conditions of $29 \mathrm{kA}$ $\mathrm{m}^{-1}$ and $520 \mathrm{kHz}^{17}$ (30 times the TV). Spherical clusters ( $60 \mathrm{~nm}$ size) of carboxymethyldextran (CMD) coated iron oxide nanoparticles of 18 $\mathrm{nm}$ dissipate $720 \mathrm{~W} \mathrm{~g}^{-1}$ when excited at $10 \mathrm{kA} \mathrm{m}^{-1}$ and $410 \mathrm{kHz}^{10}(8.2$ times the TV) and nanoflower like assemblies ${ }^{18}$ dissipating $1175 \mathrm{~W} \mathrm{~g}^{-1}$ when excited at $21.5 \mathrm{kA} \mathrm{m}^{-1}$ and $700 \mathrm{kHz}$ (30 times the TV) has also been reported. Another way to improve the SAR is by modifying MNP chemical composition, for instance by doping with $\mathrm{Zn}, \mathrm{Mn}$ or Co. We have reported SAR value of $367 \mathrm{~W} \mathrm{~g}^{-1}$ at $260 \mathrm{kHz}$ and $41 \mathrm{kA} \mathrm{m}^{-1}$ (21 times TV) using a $16 \mathrm{~nm}$ size particle with $3.3 \%$ of Fe atoms replaced by $\mathrm{Zn}^{19}$. For Co substitution ${ }^{20}, 440 \mathrm{~W} \mathrm{~g}^{-1}$ was obtained at $700 \mathrm{kHz}$ and 24.7 kA m ${ }^{-1}$ (35 times TV) and Mn substitution ${ }^{21}$ effectively leads to an increase in the MNPs heating efficiency. Interesting, using exchange coupled nanomagnets ${ }^{22}$, combining hard and soft magnetic phases like $\mathrm{CoFe}_{2} \mathrm{O}_{4} @ \mathrm{MnFe}_{2} \mathrm{O}_{4}$, a SAR value two order of magnitude larger was reported for $15 \mathrm{~nm}$ composite particle in comparison with the values for its components $\left(9 \mathrm{~nm} \mathrm{CoFe} \mathrm{O}_{4}\right.$ and $\left.15 \mathrm{~nm} \mathrm{MnFe}_{2} \mathrm{O}_{4}\right)$ at $500 \mathrm{kHz}$ and $37.3 \mathrm{kA} \mathrm{m}^{-1}$, (37 times the TV). Composition modification comparing with iron oxides entails the problem of increasing cytotoxicity.

Regarding to the effects related to the interaction between MNPs and biological entities, SAR values appear highly suppressed when MNPs are located in cells as described in various in vitro assays ${ }^{20,23-27}$. The pioneering work of Fortin et $a^{20}$ related this attenuation to the inhibition of Brown contribution to heat generation, due to abrupt change in media viscosity. Also, significant reductions in cell viability without macroscopic cell cultures temperature rise has been reported for various cancer cells with MNPs internalised and exposed to AMF, like in Hella cells loaded with silica-coated manganese oxide ${ }^{27}$ and in dendritic cells loaded with magnetite having either positive $(\mathrm{NH}+2)$ or negative $(\mathrm{COOH}-)$ surface functional groups ${ }^{28}$ as well as in the case of nanoparticles engineered for targeted hyperthermia as CMD coated magnetite conjugated with epidermal growth factor (EGF) in MDAMB-468 and MCF-7 cells ${ }^{23,26}$. More recently, De Sousa et al ${ }^{24}$ provided evidence that there was local heating even though no global temperature increase was sensed. To this end, human lung adenocarcinoma cells (A549 cell line) were transduced with a lentiviral vector encoding the expression of the enhanced green fluorescence protein (EGFP) under the action of the inducible human heat shock protein 70B promoter. These modified cells (A549HSP) that express the EGFP under thermal stress. also expressed the EGFP after being loaded with magnetite MNPs and exposed to RF fields indicating that endocyted MNPs create hot spots inside the cells, even though the amount of heat released was not enough to globally increase the cell culture temperature ${ }^{24}$. The need of distinguishing between global heating and cell-treatment efficacy to improved hyperthermia treatment was later discussed elsewhere ${ }^{25}$. Other explanation referred to aggregation inside cell endosomes ${ }^{29,30}$. Jeon et $\left.a\right|^{29}$ proposed that the formation of super-aggregates in the intracellular milieu impacts on SAR, reducing it from above $200 \mathrm{~W} \mathrm{~g}^{-1}$ for aggregates composed of fewer than 50 primary particles to below $50 \mathrm{~W} \mathrm{~g}^{-1}$ for superaggregates, and stressed that the underlying physical mechanism by which aggregation leads to reduction in SAR remains unclear. A review on nanoparticle designed for targeted hyperthermia and their interaction with cell of importance in systemic delivery can be found in Kozissnik et al. ${ }^{31}$

On the other hand, the applicability of $\mathrm{MH}$ has been proved in animal model ${ }^{32,33}$ and in clinical trials ${ }^{34}$; nevertheless the acquisition and analysis of SAR data on ex vivo tumour tissue can be barely found. Importantly, the influence of particle immobilization in tumour tissue and their influence in magnetic properties has been addressed by Dutz et $\mathrm{al}^{35}$. These authors proved by ex vivo analysis of human breast tumours grown in immunodeficient mice injected with multicore 
nanoparticles, that a temperature increase owed to Brown relaxation could be neglected due to MNPs rotation suppression. However, they registered a tissue temperature increase of about $20^{\circ} \mathrm{C}$ within the first $60 \mathrm{~s}$ in a tumour loaded with $7 \mathrm{mg}$ of multicore nanoparticle exposed to an AMF of $400 \mathrm{kHz}$ and $25 \mathrm{kA} \mathrm{m}^{-1}$.

In the present work, we attempt a holistic approach to better comprehend which are the relevant aspects that can facilitate the translation of magnetic hyperthermia to clinical uses. To that, we focus in one of the most promising type of material reported so far $^{18}$, in fabricating a portable multipurpose field applicator with therapeutic specifications and in the material and applicator validation in a tumour model. To this end, we synthesize a flower like nanostructures and proved that they satisfied biomedical requirements, we designed and built a field applicator that works with therapeutic specifications and then, we focus on the issue of tumour heating efficiency in a tumour model at field conditions close to therapeutic ones.

Nearly monodisperse citrate coated nanoclusters (NCs) of crystallographically aligned magnetite MNPs were fabricated by a coprecipitation polyol method. A meaningful structural and magnetic characterization was carried out to resolve NCs composing particles collective behaviour and to support SAR data analysis. Tumour ex vivo analysis was selected to study static tissue properties. Melanoma tumours grown in $\mathrm{C} 57 \mathrm{BL} / 6 \mathrm{~J}$ mice challenged with murine B16-F0 melanoma cells were extracted after mice sacrifice and selected as the ex vivo tumour model. The applicability and dose-response characteristic of selected NCs were ex vivo tested and compared with the SAR values of these NCs dispersed in water and agarose-gelphantoms.

\section{Results and discussion}

\section{A. Nanocluster suspensions synthesis and properties.}

Among the various types of nanostructures reported for $\mathrm{MH}$, nanoclusters composed of primary MNPs crystallographically aligned with adjacent ones are the most promising candidates to improve magnetic heating. The first method for achieving such nanocluster was presented by $\mathrm{Ge}$ et $\mathrm{al}^{36}$, who reported a protocol for the preparation of polyacrylate-capped superparamagnetic magnetite $\left(\mathrm{Fe}_{3} \mathrm{O}_{4}\right)$ clusters with tuneable sizes from 30 to $180 \mathrm{~nm}$ by one step high-temperature hydrolysis process. These NCs exhibited good stability and high dispersibility in aqueous media (water), without any further functionalization. Since the biodegradability and biocompatibility of the polyacrylic acid attached on the magnetic clusters is unclear ${ }^{37,38}$, we choose to modify a synthetic variation recently proposed by Hougouneng et $\mathrm{al}^{18}$, of citrate coated NCs. The formation and growth rate of iron oxide nanoparticles in the presence of this polyol mixture is slow and the nanoparticles have enough time to rotate and contact with neighbour nanoparticles at the angle minimizing energy. Some misalignment between particles disappear during Ostwald ripening process ${ }^{39}$.

Nanocluster size and component particle size distributions, their crystalline structure and the degree of alignment inside the nanocluster NCs were characterized by means of Transmission Electron Microscopy and Electron Diffraction. Representative HR-TEM images of the NCs are shown in Figure 1. The images corroborate that the polyol procedure, using DEG/NMDEA 1:1 (v/v), yields nearly monodisperse multi-core nanostructures. No isolated particles were observed. Size histograms were constructed by measuring and counting NCs and composing MNPs sizes from several micrographics of each sample. Histograms are shown in Figure S1 of ESI file. The so derived mean nanocluster diameter $D_{N C}$ and nanoparticle $D_{N P}$ diameter and their standard deviation (s.d) are summarised in Table 1 together with main synthesis parameters. These NCs displays mean sizes between 20 and $35 \mathrm{~nm}$. Hydrodynamic sizes $\left(D_{\text {HYD }}\right)$ of NC in aqueous suspensions range between 22 and $45 \mathrm{~nm}$ as seen in Table 1. For sample NC20 suspended in a $1 \%$ PBS solution $D_{H Y D}$ is $29 \pm 7 \mathrm{~nm}$.

Table 1. NC synthesis conditions and structural properties. $T_{i}$ is the initial temperature, $t_{s}$ is the time during which the solution was kept at $220^{\circ} \mathrm{C}, D_{N C}$ and $D_{N P}$ are nanocluster and primary particle diameters determined from HR-TEM, $D_{H Y D}$ is the hydrodynamic size, of the NC dispersed in water, deduced from Dynamical Light Scattering (DLS) number distribution.

\begin{tabular}{|c|c|c|c|c|c|}
\hline SAMPLE & $T_{i}$ & $t_{s}$ & $D_{N C} \pm$ s.d & $D_{N P} \pm s . d$ & $D_{H Y D}$ \\
$\left({ }^{\circ} C\right)$ & $(h)$ & $(n m)$ & $(n m)$ & $(n m)$ \\
\hline NC20 & 15 & 12 & $20 \pm 7$ & $9 \pm 3$ & $22 \pm 7$ \\
\hline NC26 & 17 & 13 & $26 \pm 6$ & $8 \pm 3$ & $29 \pm 10$ \\
\hline NC34 & 30 & 13 & $34 \pm 4$ & $8 \pm 2$ & $45 \pm 14$ \\
\hline
\end{tabular}

Selected area electron diffraction (SAED) pattern shown at the inset of Figure 1d was indexed with a cubic spinel structure (space group Fd3m, card \#750449). When selected area includes many NCs the image displays diffraction rings in agreement with different directions contributions of the randomly oriented NCs. When just one cluster is selected as shown in the left inset of Figure 2c, diffraction spots appear in agreement with Fourier transformation (FT at the centre of a NC with the edge of several nanoparticles) of marked areas confirming the crystallographic alignment of the MNPs forming a NC. The continuity of the crystal lattice planes across nanoparticles boundaries can be clearly observed in Figure 1b (additional TEM images are presented in Figures S2 to S7 of ESI file). In addition, the filtered FT of a NC from NC20, shown in Figure 2a, reveals that this NC is composed of primary particles exposing interplanar distance $0.26 \pm 0.03 \mathrm{~nm}$ corresponding to the (311) plane but along two orientations forming an angle of $33^{\circ}$, see Figure $2 b$.

These nanostructures to be ideal materials for biomedical application must conjugate large magnetic susceptibility with sizes within magnetic single domain range assuring superparamagnetic behaviour and consequently null magnetization at zero applied field, stability in biomedical fluids and low toxicity. 


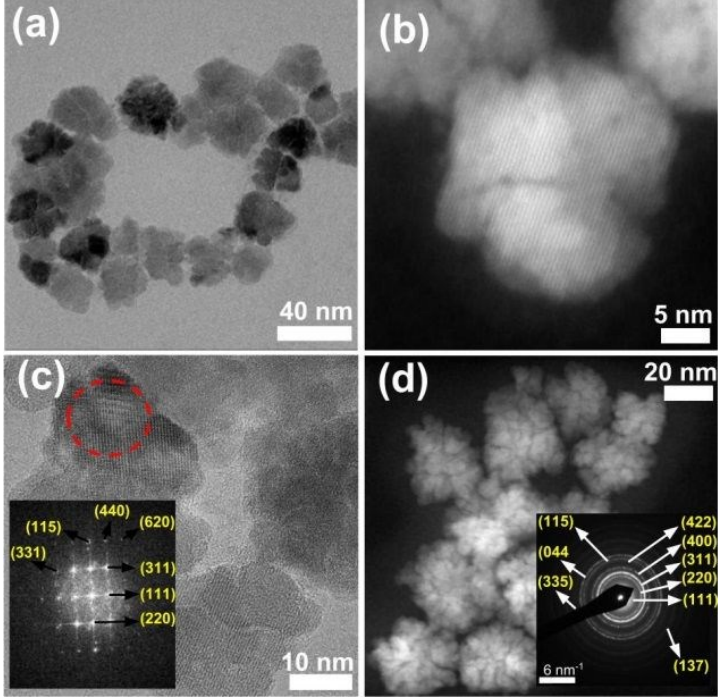

Figure 1: HR-TEM and STEM of NC26 panels (a), (b) and (c) and of NC34 (d). Insets in: (c) Fourier transformation of the areas inside the red circles, and (d) is the electron diffraction pattern including many NCs displaying rings in agreement with their random orientation. Indexed with the spinel structure.

The MNPs composing the NCs display mean sizes $\left(D_{N P}\right)$ of about $8 \mathrm{~nm}$, being clearly within single magnetic domain range. On the other hand, each NC of volume $V_{N C}$ is a collection of single domain particles of magnetic volume $V_{N P}$ and giant magnetic moment $\mu_{N P}=M_{s} \cdot \rho \cdot V_{N P}$ ( $\rho$ is the mass density for magnetite, $5175 \mathrm{~kg} \mathrm{~m}^{-3}$ ). HR-TEM data shows crystallographic alignment between MNPs, but how their magnetic moments are arranged is still not clear and we further investigated here. In a recent report, we proved ${ }^{40}$ that the data needed to predict the SAR of a magnetic colloid are the saturation magnetization $M_{s}$, mean nanoparticle volume $V_{M N P}$ and a mean activation energy $\langle U\rangle$. Where $\langle U\rangle$ includes, besides the anisotropy term equal to $K V_{M N P}$ (where $K$ is the magnetic anisotropy constant) contributions due to size and shape polydispersity, aggregation and dipolar interactions among particles. In general, an increase in polydispersity and/or aggregation tends to lower the SAR, while an increase in magnetic interactions strength results in a SAR increase. A rational choice of independent experiments allowed us to derive consistent $\langle U\rangle$ values that were successfully used to reproduce the SAR of various nanoparticle suspensions ${ }^{40}$. Following a similar methodology, in this work we first measured the isothermal magnetization field dependence at $300 \mathrm{~K}$ of the NCs in aqueous suspensions, exemplified in Figure 3a for the NC20 (see fitting details in section $\mathrm{S} 1$ in the ESI file). The $M$ vs. $H$ loop displays no coercive field in agreement with unblocked NCs performing Brownian motion.
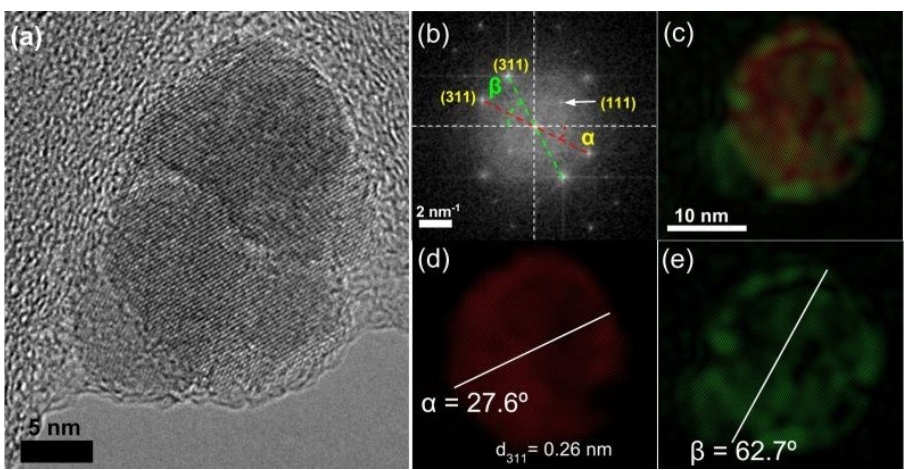

Figure 2: (a) HR-TEM of one nanocluster from NC20 and its Fourier transform (b) indexation is consistent with electron beam parallel to the [011] direction of magnetite. (c) Filtered Fourier transform showing two orientations of the same crystallographic plane, green and red colours correspond to the (311) planes forming an angle of $61^{\circ}$ and $28^{\circ}$ with horizontal axis.

The $M_{s}$ and mean magnetic moment $\mu_{M}$ were derived from the fitting of $M$ vs. $H$ curves with an Langevin type integral that continuously adds contribution from NCs of total magnetic moment distributed following a log-normal distribution, to the magnetization at each field ${ }^{41}$. Fitted parameter are number of nanoclusters $N /$ Fe mass, $\sigma_{\mu}$ standard deviation of $\ln \left(\mu / \mu_{0}\right)$, median magnetic moment $\mu_{0}$, and high field susceptibility $\chi$ included by adding a linear term $\chi \mathrm{H}$. From fitted values saturation magnetization $M_{s}=N \mu_{M}$ and the mean moment $\mu_{M}$ $=\mu_{0} \exp \left(\sigma_{\mu}^{2} / 2\right)$ were derived and are listed in Table 2 (Magnetization curves of all the samples, at $300 \mathrm{~K}$, are shown in Figure S8 of the ESI file). The so derived saturation magnetization values are close to bulk magnetite value ${ }^{42}$ of $120 \mathrm{~A} \mathrm{~m}^{2} \mathrm{~kg}_{\mathrm{Fe}}{ }^{-1}$. Only NC34 displays a low $M_{s}$ value, probably those nanoparticles bears a large magnetic dead layer. Large $M_{s}$ is one of the requirements to achieve larger SAR values ${ }^{43}$ as the SAR depends with the $M_{s}$ square $^{2}$. These $\mu_{M}$ values are in agreement with NCs size derived from TEM, being much larger than, for instance, the magnetic moment of a single $8 \mathrm{~nm}$ spherical particle whose bulk magnetic moment is 10867 $\mu_{B}$. The latter was estimated using that magnetite magnetic moment is $24 \mu_{B}$ per unit cell and a lattice constant $a=8.3963 \AA$. This analysis indicates that the NCs behave as a single magnetic domain particle shading light in a pending question of the field. If the crystallographic alignment is complete the NCs have cubic magnetic anisotropy with easy axes along [111] direction. This direction form an angle of $22^{\circ}$ with the vector $(1,3,3)$ normal to the planes $(311)$ seen in Figure 2. However, for partial alignment, as for example for the NC shown in Figure 2, where a fraction of the composing MNPs has the easy directions at $76.7^{\circ}$ relative to the vector $(1,3,3)$ normal to the planes (311) and drawn in red, and the complementary fraction an easy axis forming an angle of $33^{\circ}$ with the ease axis of the red part and also at $76.7^{\circ}$ relative to the rotated plane normal vector (green). This misalignment could lower NC total magnetic moment as compared to NC with full alignment but is still expected to be larger than that of NCs of randomly oriented MNPs for which SAR decrease ${ }^{41}$. 



Figure 3: (a) Magnetization loops measured at 5 and $300 \mathrm{~K}$ for NC20. Inset: zoom showing low field magnetization. Solid lines are for best Langevin fits, (b) Coercive field $H_{c}$ temperature dependence derived from d.c. magnetization curves. (c) Magnetization under ZFC/FC protocols using DC applied field of $4 \mathrm{kA} \mathrm{m}^{-1}$ for NC20. (d) Absolute values of the cluster magnetic moments $\left|\mu_{i}\right|$ as function of the Monte Carlo steps time (MCS) at 5 and $300 \mathrm{~K}$, being $i=x, y, z$ and $z$ coincident with anisotropy axis. Inset in $d$ shows a typical equilibrium configuration of particle magnetic moments at $t=10^{5} \mathrm{MCS}$. The particles are shrinked to allow better visualization of the arrows representing the NPs magnetic moments at $300 \mathrm{~K}$.

Then, we measured the hysteresis loop at 5K, shown in Figure 3a for NC20 and $H_{c}$ values at selected temperatures $(6,8,15,20,26 \mathrm{~K})$ shown in Figure $3 \mathrm{~b}$ for all the samples. The former was used to estimate an anisotropy field, $H_{K}=2 K / \mu_{0} M s$, of about $120 \mathrm{kA} \mathrm{m}^{-1}$ for our samples. This is an important piece of information since for magnetic hyperthermia experiments increasing the field strength amplitude $H_{0}$ to values larger than $H_{K}$ results in no further SAR improvement. The $H_{c}$ vs. $T$ behaviour was used to semi-empirically derived $\langle U\rangle$ (using equation 4 in the ESI file) and to calculate SAR using Stoner-Wohlfarth theory ${ }^{44}$ as explained elsewhere ${ }^{40}$. Values of $\langle U\rangle$ and calculated SAR $\left(S A R_{c}\right.$ ) are listed in Table 2 (for calculation details see $\mathrm{S} 2$ section of ESI file). The $S A R_{c}$ values are in good agreement with those calorimetrically determined at $100 \mathrm{kHz}$ and $9.3 \mathrm{kA} \mathrm{m}^{-1}$, also listed in Table 2 and the heating curves are shown at Figure S9 of ESI file. Importantly, we have shown that SAR of a NCs suspension can be well reproduced using a procedure valid for suspension of single domain MNPs. The FC data acquired using a DC field of $4 \mathrm{kA} \mathrm{m}^{-1}$ is exemplified in Figure $3 \mathrm{c}$ for NC20. The FC curve do not display any of the characteristics of an antiferromagnetic order ${ }^{45}$. Moreover, to get a better insight on how magnetic moments are arranged in the NCs, we performed a Monte Carlo simulation. Figure $3 d$ shows time dependence of NC magnetization in the easy direction (z-axis) and in the perpendicular directions $(x, y)$ at $5 \mathrm{~K}$ and $300 \mathrm{~K}$ for a NC composed by 14 single magnetic domain nanoparticle of $8 \mathrm{~nm}$ size. The magnetic moment projection on the anisotropy axis (z-direction) is much larger than in the perpendicular directions, i.e. magnetic moments predominantly align in the NC easy direction. A snapshot for $300 \mathrm{~K}$ simulation is shown in the inset. The difference between $\mu_{z}$ and $\mu_{\mathrm{x}, \mathrm{y}}$ becomes larger as temperature is reduced as expected for a ferromagnetic alignment.

As mentioned, to achieve stability the NCs were citrate coated. Z potential results, obtained from Laser Doppler Electrophoresis measurements, indicated $\zeta$ values were between -30 and $-44 \mathrm{mV}$ confirming that the coating was successful and the nanoclusters are negatively charged. Cytotoxicity was analysed by means of MTT (3[4,5-dimethylthiazol-2-yl]-2,5 diphenyl tetrazolium bromide) assay, based on the conversion of MTT into formazan crystals by living cells, which determines mitochondrial activity ${ }^{46}$. Results, shown in Figure $4 \mathrm{a}$, indicate that $17 \mathrm{~h}$ exposure of B16-F0 cells to $116 \mu \mathrm{g}_{\mathrm{Fe}} \mathrm{mL}^{-1}$ of citrate-coated $\mathrm{Fe}_{3} \mathrm{O}_{4}$ nanoclusters did not significantly affect cell viability.


Figure 4: (a) Cell viability derived from MTT assay, (b) and(c) show VSM loop of a pellet of B16-F0 cell culture incubated in medium doped with NCs at a concentration of $116 \mu \mathrm{g}_{\mathrm{Fe}} \mathrm{mL}^{-1}$ for $17 \mathrm{~h}$ and cryoTEM of B16-F0 cell incubated with NCs respectively(d) detail of NCs organization inside the cell endosome, (e) TEM of B16-F0 cell in the tumour in vivo $24 \mathrm{~h}$ after NC20 injection and AMF application.(f) Detail of NCs organization in the cytosol.

From the analysis a magnetic loop (VSM data) of the cell culture after incubation at a concentration of $116 \mu \mathrm{g}_{\mathrm{Fe}} \mathrm{mL}^{-1}$ shown in Figure $4 \mathrm{~b}$, an 
uptake of about $10.6 \mathrm{pg}_{\mathrm{Fe}} /$ cell was obtained. VSM data analysis used to obtained the uptake is described in Experimental section F. The uptake is the result of the NC internalization into endosomes exemplify in Figures $4 c$ and $4 d$ with TEM image (see additional B16-F0 cells TEM images in Figure S10 in the ESI file). Our in vitro results indicate that NC are not cytotoxic for B16-F0 cells and agree with those previously reported for single MNPs ${ }^{43,47}$.
Figures $4 \mathrm{e}$ and $4 \mathrm{f}$ show the NC in the tumour $24 \mathrm{~h}$ after in vivo NC injection and AMF application as described in section C. The NC distribution in cells of tumour tissue greatly differs from that shown for in vitro cell culture. Endosomes densely loaded with NCs and/or the presence of super-aggregates of NCs are absent.

Table 2. Nanocluster aqueous suspensions properties: $[x]$ is the Fe mass concentration, $\mu_{M}$ is the NC mean magnetic moment determined from the magnetization curve with saturation magnetization $M_{s}$ at $300 \mathrm{~K} .\langle U\rangle$ is the mean activation energy determined from $H c v s T$ data analysis and $S A R$ is the specific absorption rate measured at $100 \mathrm{kHz}$ and $9.3 \mathrm{kA} \mathrm{m}^{-1}$. SAR is the specific absorption rate semi-empirically calculated as explained in the text.

\begin{tabular}{|c|c|c|c|c|c|c|c|c|}
\hline SAMPLE & $\begin{array}{c}{[x]} \\
\left(\mathrm{mg}_{\mathrm{Fe}} \mathrm{mL}^{-1}\right)\end{array}$ & $\begin{array}{c}\mu_{M} \\
\left(10^{3} \mu_{B}\right)\end{array}$ & $\begin{array}{c}M s_{300 \mathrm{~K}} \\
\left(A m^{2} k^{-1}{ }_{\mathrm{Fe}}\right)\end{array}$ & $\begin{array}{c}M s_{5 K} \\
\left(A m^{2} k^{-1}{ }_{F e}\right)\end{array}$ & $\begin{array}{c}H_{K 5 K} \\
\left(k A m^{-1}\right)\end{array}$ & $\begin{array}{c}\langle U\rangle \\
\left(10^{-20} \mathrm{~J}\right)\end{array}$ & $\begin{array}{c}S A R \\
\left(W g_{F e}^{-1}\right)\end{array}$ & $\begin{array}{c}S A R_{c} \\
\left(W g^{-1}{ }_{F e}\right)\end{array}$ \\
\hline NC2O & $7.7 \pm 0.1$ & $67 \pm 2$ & $79 \pm 2$ & $95 \pm 1$ & 121 & $2.8 \pm 0.1$ & $5.4 \pm 0.2$ & 7.2 \\
\hline NC26 & $10.4 \pm 0.2$ & $70 \pm 2$ & $54 \pm 2$ & $76 \pm 2$ & 159 & $2.3 \pm 0.1$ & $12.2 \pm 0.6$ & 9.8 \\
\hline NC34 & $3.6 \pm 0.1$ & $33 \pm 1$ & $39 \pm 1$ & $48 \pm 1$ & 126 & $6.2 \pm 0.3$ & $11.5 \pm 0.6$ & 10.3 \\
\hline
\end{tabular}

\section{B. Specific absorption rate measurements.}

Once proved that the NCs fulfilled biomedical requirements we analysed their heating efficiency in melanoma tumour tissues, where the NCs unavoidable distribute heterogeneously after injection and compare with the heating efficiency in homogenous NCs dispersions achieved in agarose gel and liquid. As we already noted in the introduction it is extremely important to carry out these analysis at field frequency and amplitude within biomedical safe range. With the aim of using the same device (without modifications) for phantoms, in vitro and in vivo, we designed and built a portable applicator that can hold large samples and works at $100 \mathrm{kHz}$ and field amplitudes that can be continuously varied between 2 and $15 \mathrm{kA} \mathrm{m}^{-1}$ without watercooling, i.e. at the same frequency and field amplitude range used for clinical trials ${ }^{12}$. Applicators previously designed for $\mathrm{MH}$ either are bulky or only useful for small samples. Some use a resonant network with a coil made of hollow-copper-pipe refrigerated with water ${ }^{48-51}$ or use Litz wire with ferromagnetic core achieving the desired uniform field in a reduced volume ${ }^{52-54}$ not useful for in vivo experimentation or for large samples. None of them end up with our portable design (shown in figure 5) that uses parallel LLC resonant network with Litz wire coil and forced air convection cooling and can be transported without requiring specialized installation ${ }^{55}$. Front and side views of the field applicator are shown in Figure 5, where the main components are referenced. Field was continuously sensed during field applications using single turn sensing coil and calibration was achieved with a secondary coil. A full description is placed at the ESI file.

A major challenge when dealing with large samples concerns to field uniformity. In Figure 6 we show a field amplitude simulation over the whole receptacle volume. Due to coil finite length, field variations across the central area relative to the coil centre, where the tumour is placed for field applications, for $\pm 7.5 \mathrm{~mm}$ in radial direction results in $+1.89 \%$ field variation while in the coil exe direction, variations of $\pm 7.5 \mathrm{~mm}$ results in $-1.45 \%$ field variation. For measurements the samples are carefully placed in the centre of the coil and the whole tumour volume (15 $\mathrm{mm}$ in diameter) is uniformly affected by the field. 

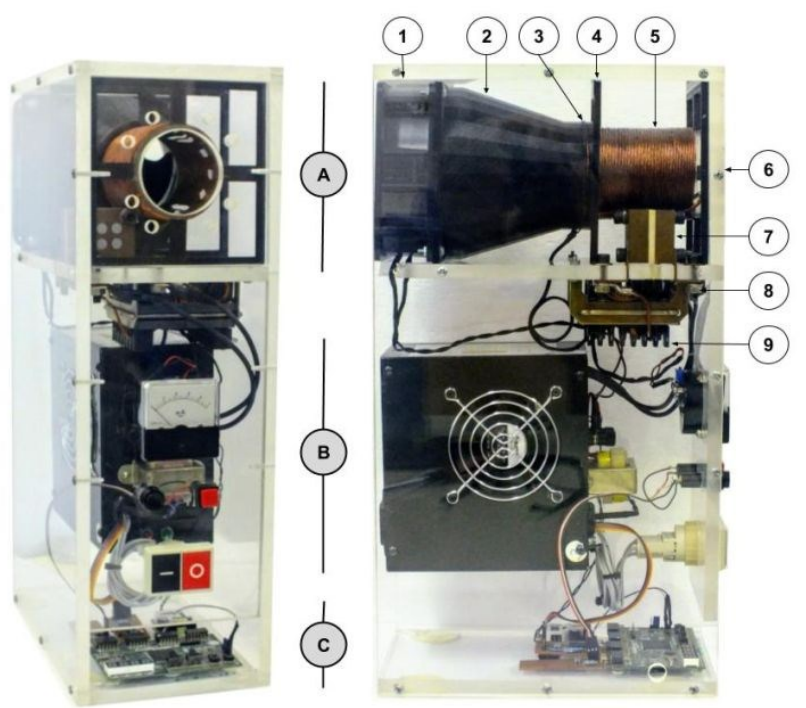

Figure 5: Front and side view of mechanical and electronic parts of the portable AMF field applicator. Main sections: A) high voltage section, B) AC/AC converter (rectifier and inverter), control panel and indicators C) CPLD based digital modulator for frequency and amplitude control. Resonant circuit and cooling: 1) 5" AC fan, 2) air nozzle, 3) single turn sensing coil, 4) plastic mounting brackets, 5) resonant inductor, 6) receptacle, 7) resonant capacitor, 8) copper bars and 9) serial inductor.



Figure 6: Field amplitude simulation for a Litz wire coil of $30 \mathrm{~mm}$ radius and $76 \mathrm{~mm}$ length. The circle in the centre stands to represent a $15 \mathrm{~mm}$ tumour. In red colour support holding a secondary coil used to calibrate the device.

Time-dependent temperature curves of NCs aqueous suspensions, agarose gel dispersion and ex vivo tumours were determined by calorimetric measurements at $100 \mathrm{kHz}$ and $9.3 \mathrm{kA} \mathrm{m}^{-1}$ using the portable applicator described. The SAR values of the magnetite nanoclusters aqueous dispersion are listed in Table 2 (see the heating curves in Figure S9 in the ESI file). Notice that for similar size NC of maghemite a value of $1175 \mathrm{~W} \cdot \mathrm{g}^{-1}$ was reported ${ }^{18}$ at $21.5 \mathrm{kA} \cdot \mathrm{m}^{-1}$ and $700 \mathrm{kHz}$. Scaling that SAR value to our field condition, it results in $\sim 36$ $\mathrm{W} \mathrm{g}^{-1}$ i.e. in the same order of magnitude of our NCs.

Next, agarose gels phantoms were prepared by adding $1 \%(\mathrm{~m} / \mathrm{V})$ of agarose to $700 \mu \mathrm{L}$ aqueous suspension after concentration, to immobilize the NCs in the milieu, approaching tumour viscosity and inhibiting Brown contribution to heat generation. The so-prepared samples consist of NCs uniformly distributed in the agarose gel. The measured heating curves are shown in Figure 7a. Based on these results, NC20 and NC34 were selected for the ex vivo hyperthermia experiments. The amount of NCs to inject into the tumours, expressed as the mass of $\mathrm{Fe}\left(m_{\mathrm{Fe}}\right)$, was estimated from agarose gel temperature increase.


Figure 7: Heating curves of NCs measured in the portable applicator at $100 \mathrm{kHz}$ and $9.3 \mathrm{kA} \mathrm{m}^{-1}$ in a) homogenously dispersed in $700 \mu \mathrm{l}$ of agarose-gel-phantoms and b) in tumour tissue, displayed as temperature increase relative to control. c) Heating curve of NC34 in tumour tissue at $265 \mathrm{kHz}$ and $40 \mathrm{kA} \mathrm{m}^{-1}$. d) Thermal image of heated tumour after recording the heating curve shown in b). Inset in $d$ : thermal image of a tumour injected with $10 \mathrm{mg}_{\mathrm{Fe}}$ of NC34, no heating was observed after RF application with optical fiber (panel b) nor with thermal image.

To evaluate NC20 heating efficiency in tumour tissue, experiments were performed with 7 mice. 4 mice received two intratumoural injections, two hour spaced, of $100 \mu \mathrm{L}$ of NC20 in PBS (a total of $55 \mathrm{mg}$ of Fe), whereas tumours from 3 mice received PBS (control group). After $24 \mathrm{~h}$ the mice were sacrificed and tumours were exposed to the AMF. A temperature increase of $7.4 \pm 1.4{ }^{\circ} \mathrm{C}$ was accomplished within 
the first $23 \mathrm{~min}$ in NC20 treated-mice (see Table 3 and Figure7b). No significant temperature increasing $(\Delta T)$ was observed in control group. Experiments with NC34 were performed in 12 mice (8 injected with NC34 suspended in PBS and 4 controls only with PBS).Initially, two mice were injected with $3.0 \mathrm{mg}_{\mathrm{Fe}}$ of NC34 i.e. the amount of Fe that had produced a $\Delta T$ of about $10^{\circ} \mathrm{C}$ in $700 \mu \mathrm{L}$ of agarose gel (see Figure 7a). Surprisingly, no temperature increase was achieved in these tumours (see Figure 7b). Notice also that tumour volumes were smaller than the gel volume. Then, a third one received $6 \mathrm{mg}_{\mathrm{Fe}}$ and four other mice received $10 \mathrm{mg}_{\mathrm{Fe}}$ (see Table 3). Extracted tumours were exposed to the AMF and again no $\Delta T$ increase was achieved.

Then, to control the magnetic material, a suspension of NC34 in PBS at a concentration of $50 \mathrm{mg}_{\mathrm{Fe}} \mathrm{mL}^{-1}$ was exposed to the AMF as well as a dilution $1 / 12\left(4.2 \mathrm{mg}_{\mathrm{Fe} \mathrm{mL}} \mathrm{mL}^{-1}\right)$ resulting in $\Delta T$ of $8{ }^{\circ} \mathrm{C}$ and $6.5^{\circ} \mathrm{C}$ respectively (not shown).

Based on these results, one last mouse was injected with $55 \mathrm{mg}_{\mathrm{Fe}}$ of NC34. A $\Delta T$ of $8^{\circ} \mathrm{C}$ was accomplished within the first $29 \mathrm{~min}$ (see Table 3 and Figure $7 \mathrm{~b}$ ). Thermal images were acquired with infrared (IR) camera immediately after field irradiation, an example is shown in Figure $7 \mathrm{~d}$ for a tumour injected with $200 \mu \mathrm{l}$ of NC34 suspension (55 $\left.\mathrm{mg}_{\mathrm{Fe}}\right)$. IR camera image the whole tumour surface detecting IR waves from a penetration depth of about $3 \mathrm{~mm}^{56}$. A full agreement between fiber optic and IR camera measurements were obtained for all the tumours. These experiments show that $2.5 \mathrm{mg}_{\mathrm{Fe}}$ of NC34 dispersed in agarose $\left(700 \mu \mathrm{L}\right.$ of $\mathrm{NC3} 4$ at $\left.3.6 \mathrm{mg}_{\mathrm{Fe}} \mathrm{mL}^{-1}\right)$ induced a temperature increase of $10^{\circ} \mathrm{C}$ but $10 \mathrm{mg}_{\mathrm{Fe}}$ of the same magnetic material induce no detectable temperature elevation in a tumour tissue (inset Figure 7d). Even more, SAR value of NC20 aqueous suspension, see table 2, is around half of that of NC34 aqueous suspension, however near the same temperature increase is obtained in tumour tissue when injected with $55 \mathrm{mg}_{\mathrm{Fe}}$, indicating that there is no clear correlation between fluid and gel SAR and $\Delta T$ values determined in a heterogeneous tumour tissue. It is worth mentioning that we have measured the heat capacity of both materials, being $4.3 \pm 0.1 \mathrm{~J} \mathrm{~g}^{-1{ }^{\circ}} \mathrm{C}^{-1}$ for $1 \%$ agarose gel and $4.2 \pm 0.1 \mathrm{~J} \mathrm{~g}^{-1}{ }^{\circ} \mathrm{C}^{-1}$ for tumour tissue.

Table 3: Ex vivo assays results. $m_{F e}$ is the mass of Fe contained in the volume of NCs injected in each tumour, $[X]_{i n}$ is the injected concentration, $V_{\text {tum }}$ is the volume of each extracted tumour exposed to the AMF, $\Delta t$ is the time required to reach the highest tumour temperature, $\Delta T$ represents the maximal increase of temperature with respect to a non-injected NCs tumour (control).

\begin{tabular}{|c|c|c|c|c|c|c|}
\hline$N C$ & Mouse & $\begin{array}{c}m_{F e} \\
(m g)\end{array}$ & $\begin{array}{c}{[X]_{\text {in }}} \\
\left(m g \mathrm{~mL}^{-1}\right)\end{array}$ & $\begin{array}{c}V_{\text {tum }} \\
\left(\mathrm{mm}^{3}\right)\end{array}$ & $\begin{array}{c}\Delta t \\
(\min )\end{array}$ & $\begin{array}{l}\Delta T \\
\left({ }^{\circ} \mathrm{C}\right)\end{array}$ \\
\hline \multirow{5}{*}{$\begin{array}{c}\text { NC20 } \\
\text { PBS }\end{array}$} & 1 & 55 & 275 & 150 & 27 & 5.5 \\
\hline & 2 & 55 & 275 & 250 & 22 & 7.2 \\
\hline & 3 & 55 & 275 & 250 & 23 & 8.5 \\
\hline & 4 & 55 & 275 & 211 & 21 & 8.2 \\
\hline & $\begin{array}{l}\text { mean } \\
\text { value }\end{array}$ & 55 & 275 & $215 \pm 47$ & $23 \pm 3$ & $7.4 \pm 1.4$ \\
\hline \multirow{4}{*}{$\begin{array}{l}\text { NC34 } \\
\text { PBS }\end{array}$} & $1-2$ & 3 & 32 & $245-384$ & - & - \\
\hline & 3 & 6 & 32 & 384 & - & - \\
\hline & $4-7$ & 10 & 32 & $320-352$ & - & - \\
\hline & 8 & 55 & 275 & 419 & 29 & 8 \\
\hline \multicolumn{2}{|c|}{ NC20 Agarose gel } & 51.8 & & 700 & 22 & 16 \\
\hline \multicolumn{2}{|c|}{ NC34 Agarose gel } & 2.5 & & 700 & 22 & 10 \\
\hline
\end{tabular}

Next, to be certain that the NCs are still in the tumours and to compare with other authors results, the tumours were measured at a high field of $265 \mathrm{kHz}$ and $40 \mathrm{kA} \mathrm{m}^{-1}\left(H_{0} f=106 \times 10^{8} \mathrm{~A} \mathrm{~m}^{-1} \mathrm{~s}^{-1}\right)$. Heating curves of the tumours injected with PBS suspension of NC34 are shown in Figure 7c. At this high field, the heating was too fast and the higher the mass of NCs injected the faster the heating. For instance, a tumour injected with $55 \mathrm{mg}_{\mathrm{Fe}}$ of NC34 increase $22.4^{\circ} \mathrm{C}$ in $20 \mathrm{~s}$ while 55 $\mathrm{mg}_{\mathrm{Fe}}$ of $\mathrm{NC20}$ increase $18.4{ }^{\circ} \mathrm{C}$ in $20 \mathrm{~s}$ (not shown). Other authors results ${ }^{35}$ indicated a temperature increase of about $20{ }^{\circ} \mathrm{C}$ within the first $60 \mathrm{~s}$ in a tumour, grown with MDAMB-231 cells, loaded with $7 \mathrm{mg}$ of multicore nanoparticle (composed of randomly oriented MNPs) when exposed to a high AMF of $400 \mathrm{kHz} 25 \mathrm{kA} \mathrm{m}^{-1}\left(H_{0} f=100 \times 10^{8} \mathrm{~A}\right.$ $\left.\mathrm{m}^{-1} \mathrm{~s}^{-1}\right)$. Our experiments have shown that tumours injected with 10 $\mathrm{mg}_{\mathrm{Fe}}$ of $\mathrm{NC} 34$ increased $10^{\circ} \mathrm{C}$ in $60 \mathrm{~s}$ at high field, indicating that our NCs pairs in quality other already characterized at high field.

An excised tumour of those grown from B16-F0 cells is shown in Figure 8. The tumour structurally consists of connective extracellular matrix, blood vessels and malignant cells, displaying thus large heterogeneities. When the NC suspension is intratumorally injected in vivo, the living tissue drives the NC distribution that results in an irregular NC distribution, where the NCs can end up in the interstitial extracellular matrix and in organelles such as endosomes, mitochondria and melanosomes.



Figure 8: Typical excised tumour.

Figure 9, shows TEM images of control tumours in row a) and injected with $55 \mathrm{mg}_{\mathrm{Fe}}$ of NC20 in row (b), both after AMF applications. In row a) the preservation of cellular and intracellular structures is observed. In Row b) fragmented nuclei and NCs dispersed in the cytosol and also in melanosomes are observed. 

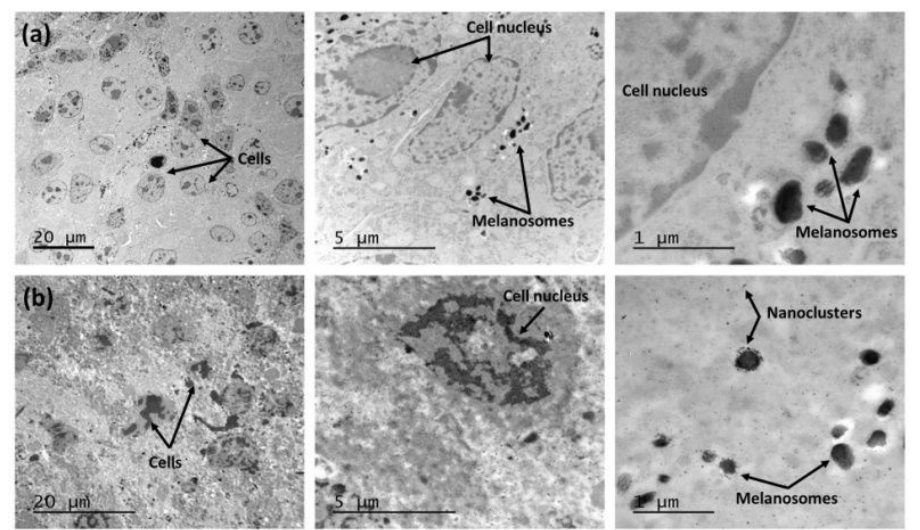

Figure 9: TEM images of B16-F0 cell tumours. Row (a) control tumour and row (b) tumour $24 \mathrm{~h}$ after injection with $55 \mathrm{mg}_{\mathrm{Fe}}$ of $\mathrm{NC} 20$ and ex vivo AMF of $9.3 \mathrm{kA} \mathrm{m}^{-1}$ and $100 \mathrm{kHz}$ application.

Ex vivo evaluation of tumour hyperthermia left aside suppression of nanostructure effective heating due to perfusion and local thermoregulation, as well as positive adjuvant effects as cell killing and tumour regression due to possible immune response induction ${ }^{57}$ and negative ones like the thermo-resistance phenomenon due to the induction of heat shock proteins (HSP) expression ${ }^{58}$. Our experiment only sense the ex vivo heating arising from the NCs distributed in tumour tissue as result from its interaction with living tissue. Results indicate a suppression of magnetic material effective heating efficiency which is not due to a change in viscosity and Brown mechanism inhibitions nor to aggregation phenomena inside endosomes as proposed in cell cultures $20,29,59$. As it was previously demonstrated in cancer cell cultures $23,24,26,27$ we hypothesized that the irregular distribution of NC inside an heterogeneous tumour generates hot spots that are responsible for a local heating but no for a global temperature increase.

To understand whether or not this affects the therapeutic outcome further experiments are needed. It is worth mentioning that it was recently shown that cell death induced in vivo on colon tumours after application of $\mathrm{MH}$ treatment occurred without a significant global temperature increase ${ }^{60}$.

\section{Conclusions}

We have ex vivo tested in tumour tissue the heating efficiency of nanoclusters composed of iron oxide magnetite nanoparticles crystallographically aligned. We found in tumour tissue a suppression of magnetic material effective heating efficiency and show that this inhibition is not due to Brown mechanism inhibition nor to NCs aggregation, instead it is related to the irregular magnetic material distribution inside the tumour. In fact, dosage had to be much increased over the values predicted from aqueous ferrofluid or agarose-gel-phantoms SAR data to achieve the same temperature increment.

We have shown that a SAR value of about $1 \mathrm{~kW} \mathrm{~g}^{-1}$ could not be accomplished at therapeutic field conditions in an ex vivo real tumour but very fast and large temperature increase was measured using high fields far off the recommended values. A word of caution should be given to studies reporting hyperthermia experiments not using therapeutically field conditions or unreal experimental settings because at these conditions non-specific heating appears due to Eddy currents. We stress the need of further integrated studies on magnetic nanostructure and tumour environment interactions that may give insight for cancer therapy.

\section{Experimental Section}

\section{A. Chemical synthesis.}

All chemical reagents used were high grade and were used without further purification. Iron(II) chloride tetra hydrate $\left(\mathrm{FeCl}_{2} \cdot 4 \mathrm{H}_{2} \mathrm{O}, 99 \%\right)$, Iron(III) chloride hexahydrate $\left(\mathrm{FeCl}_{3} \cdot 6 \mathrm{H}_{2} \mathrm{O}, 99 \%\right)$, diethylene glycol (DEG, 99\%), N-methyldiethanolamine (NMDEA, 99\%) were purchased from Sigma-Aldrich and sodium hydroxide $(\mathrm{NaOH}, 99.99 \%)$, sodium citrate tribasic dihydrate (98\%), and nitric acid $\left(\mathrm{HNO}_{3}, 70 \%\right)$ from Biopak and Ultrapure agarose from Invitrogen.

Nanoclusters composed of various $\mathrm{Fe}_{3} \mathrm{O}_{4}$ nanoparticles (core) were prepared using $\mathrm{Fe}(\mathrm{II})$ and $\mathrm{Fe}(\mathrm{III})$ precursors in polyol mixture (DEG/NMDEA) via a method adapted from Hugounenq et al. In a typical procedure $1.082 \mathrm{~g}(4 \mathrm{mmol})$ of $\mathrm{FeCl}_{3} \cdot 6 \mathrm{H}_{2} \mathrm{O}$ and $0.398 \mathrm{~g} \mathrm{(2}$ mmol) of $\mathrm{FeCl}_{2} \cdot 4 \mathrm{H}_{2} \mathrm{O}$ were completely dissolved in $80 \mathrm{~g}$ of a liquid mixture of DEG and NMDEA with 1:1 (v/v) ratio at an initial temperature $T_{j}$. The solution was stirred for $1 \mathrm{~h}$. Separately, a mixture of $0.64 \mathrm{~g}$ ( $16 \mathrm{mmol})$ of $\mathrm{NaOH}$ with $40 \mathrm{~g}$ of polyols was prepared. This solution was added to the solution of iron chlorides, and the resulting mixture was stirred for 3 more hours. Then, the temperature was raised to $220{ }^{\circ} \mathrm{C}$ at a heating rate of $\sim 3{ }^{\circ} \mathrm{C} \mathrm{min}-1$, and the mixture kept at this temperature under $\mathrm{N}_{2}$ reflux and magnetic stirring for 12-13 h. Then, the heating mantle was switched off and the mixture slowly cooled to room temperature. The black sediments were magnetically separated and washed with a mixture of ethanol and ethyl acetate $(1: 1, v / v)$ several times to eliminate organic and inorganic impurities. Citrate anions were grafted to the surfaces of the so -produced nanoclusters by adding $0.3 \mathrm{~mol}$ of sodium citrate per $1 \mathrm{~mol}$ of iron element and kept at $60^{\circ} \mathrm{C}$ for $90 \mathrm{~min}$. In order to eliminate citrate anions excess, the black precipitate was magnetically separated by means of a permanent magnet and centrifuged at $8000 \mathrm{rpm}$ for 5 minutes, the supernatant was separated and black sediments were redispersed in distilled water. $T_{i}$ was varied from 15 to $30^{\circ} \mathrm{C}$.

Chemical volumetric analysis, with $\mathrm{K}_{2} \mathrm{Cr}_{2} \mathrm{O}_{7}$ used as titrant, was used for colloid concentration [ $\mathrm{x}$ ] determination ${ }^{43}$, expressed as Fe mass per solvent volume with an accuracy of $2 \%$. [x] values were used to calculate SAR values from the heating curves obtained with calorimetric experiments, to normalize magnetic measurements and used as starting point to estimate the optimal Fe concentration to be injected in tumour tissue.

Agarose gels were prepared by adding $7 \mathrm{mg}$ of agarose in $700 \mu \mathrm{L}$ of aqueous suspension. The temperature of the mixture was increased up to boiling point and finally was cold down to room temperature.

\section{B. Structural and physicochemical properties.}

TEM measurements were carried out with several microscopes: 120 kV JEM-1400, 120 kV JEOL 1210, 200 kV JEM 2011,200 kV JEM JEOL 2100F, $200 \mathrm{kV}$ JEM-2011 and TEM TALOS F200X. To this end, a drop of colloid was dried on carbon coated copper grids. The mean diameter and polydispersity of each system were determined by counting and 
sizing over 300 particles from TEM images using ImageJ software and fitting a Gaussian distribution to the resulting particle size histograms. Samples for cryo-transmission electron microscopy (Cryo-TEM) were prepared as follows: $3 \mu \mathrm{L}$ of $\mathrm{NC}$ suspension were placed onto a Quantifoil ${ }^{\circledR}$ grid where a perforated foil was used to bear an ultra-thin carbon support foil to minimize the total specimen thickness. The drop was blotted with filter paper and the grid was quenched rapidly into liquid ethane to produce vitreous ice, avoiding the formation of crystals. The grid was then transferred into the TEM microscope, where the temperature was kept under $-140^{\circ} \mathrm{C}$ by the use of liquid nitrogen during the imaging.

Hydrodynamic diameter $\left(D_{H}\right)$ was retrieved from Dynamical Light Scattering (DLS). Colloidal system stability is analysed from Z-potential (द) values obtained from Laser Doppler Electrophoresis measurements. A Malvern Zeta Sizer Nano ZS device equipped with a He/Ne $633 \mathrm{~nm}$ laser was employed. Dispersions of magnetic NCs in Milli $Q$ water were prepared to a dilution of $1 / 60$ of the nominal concentration; $\mathrm{pH}=7.0$ to measure $\zeta$. The same dispersions were ultrasonicated to measure the average Hydrodynamic sizes $(\mathrm{nm})$. Care was taken that the DLS peak position and width were the same after three consecutive runs of 15 scans each run for all the nanoparticles. Number distribution data is reported.

\section{Static magnetic measurements.}

Magnetization as a function of applied magnetic field $(H)$ at room temperature was obtained using vibrating sample magnetometer (VSM) Lake Shore 7404 operated with maximum applied fields $\mu_{0} \mathrm{H}_{\max }=$ $1.8 \mathrm{~T}$ and/or using a superconducting quantum interference device (SQUID) magnetometer choosing $\mu_{0} H$ max $=2.0 \mathrm{~T}$. The later was also used to achieve hysteretic magnetization loops at various temperatures between 5 and $36 \mathrm{~K}$ and $M$ vs. $T$ dependence under zero field cool (ZFC) and field cool (FC) protocols with $H_{D C}=4 \mathrm{kA} \mathrm{m}^{-1}$. Magnetic measurements were performed on liquid or frozen colloids depending on the measurement temperature. A $50 \mu \mathrm{L}$ colloidal suspension were sealed in a heat shrinkable tube to prevent sample evaporation and spills.

\section{Magneto calorimetric measurements.}

To determine SAR, NCs suspension $(500 \mu \mathrm{L})$ were hold in a clear glass Dewar, agarose gel dispersion in an Eppendorf and tumours in similar plastic holder. All the experiments were carried out in non-adiabatic condition in the portable applicator is described in Section B.

Water refrigerated field generator was used for high field experiment It consist of a resonant RLC circuit Hüttinger (2.5/300) with a water refrigerated 6 turns coil of $2.5 \mathrm{~cm}$ diameter with the capability of working at different frequencies up to $265 \mathrm{kHz}$ and maximum field of $40 \mathrm{kA} \mathrm{m}^{-1}$, changing capacitors and inductances.

For AMF application the samples were centred in the coil. The temperature was sensed during the whole experiment with an optical fiber sensor placed in the centre of the sample. The sensor was connected to a calibrated signal conditioner (Neoptix) with an accuracy of $\pm 0.1{ }^{\circ} \mathrm{C}$. IR thermal imager Testo 870-1, of thermal sensitivity $<0.1{ }^{\circ} \mathrm{C}$ at $30{ }^{\circ} \mathrm{C}$, was also used for ex vivo essays. The tumours were quickly removed from the coil and thermal images were obtained immediately after field irradiation. The slope $d T / d t$ of the linear part of the heating curves enable to deduce the specific absorption rates (SAR)

$$
\text { SAR }=\frac{C d T}{[x] d t}
$$

$C$ is the volumetric heat capacity and $[\mathrm{x}]$ the concentration. Tumour tissue and agarose heat capacity was measured using modulateddifferential scanning calorimetry.

\section{E. Monte Carlo simulation.}

To analyse magnetic moments alignment in the NCs, Monte Carlo simulation was carried out using the Stoner-Wohlfarth model ${ }^{44}$ including magnetic dipolar interactions and thermal fluctuations ${ }^{61}$ in absence of external magnetic field. The sample consist on a set of 10 NCs formed by 14 spherical monodisperse $\left(D_{N P}=8 \mathrm{~nm}\right)$ single domain nanoparticles crystallographically aligned. The compactness condition and excluded volume were secured. Each particle was characterized by a magnetic moment $\vec{\mu}_{i}$ and uniaxial anisotropy $\vec{K}=K . \hat{k}$ directed along $z$ axis. The distances between NCs were set to be large enough in ordet to disregard the NCs interactions with respect to the intracluster particle ones. The system time evolution was carried out using the Metropolis algorithm ${ }^{62,63}$ from a random initial magnetic moments configuration up to reach the thermal equilibrium, with open boundary conditions and the solid angle restriction proposed by Nowak $^{63}$. The time unit, i.e. a Monte Carlo step, was defined a $\mathrm{N}$ attempts to update the magnetic moment of a randomly chosen particle, with $\mathrm{N}$ the total number of the particles in the system ${ }^{64}$. The results were averaged over 1000 realizations.

\section{F. In vitro assays, animal model and ex vivo heating experiments.}

\section{Cell line and culture conditions, viability and uptake.}

B16-F0 (murine melanoma, ATCC CRL-6322) were grown in RPMI-1640 (Gibco BRL, Gaithersburg, MD, USA) supplemented with $10 \%$ fetal bovine serum (FBS), $2 \mathrm{mM} \mathrm{L-glutamine,} 50 \mathrm{U} \mathrm{mL}^{-1}$ penicillin and $50 \mathrm{mg}$ $\mathrm{mL}^{-1}$ streptomycin.

The cytotoxicity assay ${ }^{46}$ of the NCs was performed with a MTT (3-[4,5dimethylthiazol-2-yl]-2,5 diphenyl tetrazolium bromide) on B16-F0 cell line.

The cells were seeded in a 96-well plate at density of $1 \times 10^{5}$ cells per well in $100 \mu \mathrm{L}$ of RPMI medium supplemented with $10 \%$ of fetal bovine serum(FBS) and incubated at $37{ }^{\circ} \mathrm{C}$ in a humidified $5 \% \mathrm{CO}_{2}$ incubator. When cells reach $90 \%$ of confluence, the medium was removed and replace by medium doped with $116 \mu \mathrm{g}_{\mathrm{Fe}} \mathrm{mL}^{-1}$ concentration of NCs and incubated for 17 hours. Culture protocol was chosen with the criteria of avoiding cell culture to reach dead phase. After $17 \mathrm{~h}$ of incubation, the wells were washed with sterile warm PBS to remove all NC residues and replaced with $100 \mu \mathrm{L}$ of fresh RPMI $10 \%$ FBS containing $10 \mu \mathrm{L}$ of MTT.

After incubation time, the medium was removed and $100 \mu \mathrm{L}$ of $0.04 \mathrm{~N}$ $\mathrm{HCl}$ in isopropanol was added to all wells and mixed to dissolve the formed violet crystals. After 30 minutes of incubation, cell viability was determined by measuring absorption at $595 \mathrm{~nm}$ in Amersham Bio- 
Sciences Biotrak II plate reader. Results represent mean \pm S.E.M. of three independent experiments.

For magnetometry of the cell cultures, a protocol previously described by Mazuel et al ${ }^{65}$ with modifications was followed. Briefly; B16-F0 cells were cultured and incubated with $\mathrm{NC}$ in a $25 \mathrm{~cm}^{2}$ flask. After incubation, medium was removed, and cell monolayer was washed three times with warm $1 \times$ PBS. Cells were detached from the flask and centrifuged at $300 \mathrm{xg}$ for 5 minutes. Cell pellet was resuspended with $1 x$ PBS and cells number $\left(N_{\text {cell }}\right)$ were counted in Neubauer chamber. After that, cells were fixed for 15 minutes at room temperature with $4 \%$ paraformaldehyde and then washed again three times with $1 \times$ PBS. The fixed cells (more than $1 \times 10^{6}$ cells to satisfy VSM sensitivity) were sealed in capsules and magnetization vs. applied field curves were obtained at $300 \mathrm{~K}$ by using vibrating sample magnetometer (VSM) Lake Shore 7404 operated with maximum applied fields $\mu \mathrm{OHmax}=1.8$ $\mathrm{T}$ to obtain the magnetic moment at saturation $\left(\mu_{c}\right)$ of the cell culture. Then, uptake was obtained as:

$$
\text { up }- \text { take }=\frac{\mu_{c}}{N_{\text {cell }} M_{s}}
$$

Where $M s$ is the saturation magnetization of the internalized NCs.

\section{Ex vivo studies.}

All experiments were carried out in accordance with the National Institute of Health (NIH) Guide for the Care and the Use of Laboratory Animals, and approved by the institutional animal care and use committee (CICUAL) of the School of Pharmacy and Biochemistry, University of Buenos Aires. Female C57BL/6J mice were housed under controlled conditions and were routinely used at 8-10 weeks old (approximate weight: 20-25 g). Food and water were administered ad libitum. In order to determine the increase of temperature induced by nanocluster suspension administration, $1 \times 10^{5}$ of B16-F0 cells diluted in $100 \mu \mathrm{L}$ of RPMI were injected subcutaneously in the right flank of each mouse. 10-12 days after cell inoculation, tumour volume $\sim 100 \mathrm{~mm}^{3}$, a group of mice received two or more intratumoural injections of $100 \mu \mathrm{L}$ of NC suspended in PBS at concentrations adjusted to achieve the desire amount of Fe. Control mice group received a PBS injection. After $24 \mathrm{~h}$, mice were anesthetized via i.p. with $80 \mathrm{mg}$ ketamine and $10 \mathrm{mg}$ xylazine per $\mathrm{kg}$ of body weight, and tumours were excised and submitted to an alternate magnetic field. Temperature register was performed as described in Experimental section $D$. Tumour sizes were measured with a callipers and tumour volumes were calculated using the formula: $V=\left(D \times d^{2}\right) / 2$, where $D$ is the larger diameter and $d$ is the smaller.

For Transmission Electron Microscopy analysis tumours were cut into $1 \mathrm{~mm}^{3}$ pieces after excision and fixed with $2 \%$ glutaraldehyde for two $\mathrm{h}$ and wash with phosphate buffer, post-fixed with $1 \%$ osmium tetroxide for $2 \mathrm{~h}$ at $4{ }^{\circ} \mathrm{C}$. Then, samples were gradually dehydrated in ethanol and finally acetone, and embedded in epoxy resin for $36 \mathrm{~h}$ at 35,45 y $60{ }^{\circ} \mathrm{C}$ for polymerization. Thin sections $(70 \mathrm{~nm})$ were cut using an ultramicrotome. The examination was performed with a JEM-1400 (JEOL USA, Pleasanton, CA, USA) transmission electron microscope equipped with an ES1000W Erlangshen charge-coupled device camera (Model No. 785; Gatan, Pleasanton, CA). 


\section{Journal Name}

\section{ARTICLE}

\section{Acknowledgements}

This research was funded by CONICET (PIPs 897, 154, 524 and 567), UNLP X11/680 and X11/7884, and UBACYT 20020130100024 grants of Argentina and also partially funded by the Spanish Ministry of Economy (MAT2015-64442-R and SEV2015-0496 projects, co-funded with European Social Funds). We acknowledge, O. Moscoso-Londoño for SQUID data acquisition and M. Knobel for the use of Instituto de Física 'Gleb Wataghin', Universidade Estadual de Campinas (UNICAMP) magnetometry instrumentation, R. Peralta for her extreme care with cell biological sample preparation for TEM, the Brazilian Nanotechnology National Laboratory (LNNano) for the use of cryo-TEM (project: ME-22346) facilities, Y-TEC S.A. for the use of TEM TALOS F200X under the supervision of A. Floridia and A. Caneiro and F.H. Sánchez, G. Pasquevich and P. Mendoza Zélis for useful discussions during field inductor building. Monte Carlo simulation were performed in UnCaFiyQT-INIFTA-SNCAD. M.B.F.v.Raap, P. C. S. Avruj, L. Rogin, A. Veiga, E. Spinelli, V.Blank, G.P. Saracco and M.A Bab are members of CONICET, and P.A. Soto is a fellow of CONICET, Argentina.

\section{Notes and references}

A. Jordan, R. Scholz, K. Maier-Hauff, M. Johannsen, P. Wust, J. Nadobny, H. Schirra, H. Schmidt, S. Deger, S. Loening, W. Lanksch and R. Felix, J. Magn. Magn. Mater., 2001, 225, 118126. R. E. Rosensweig, J. Magn. Magn. Mater., 2002, 252, 370374.

3 W. S. Chang, Cancer Res., 1984, 44, 4721S-4730S.

4 A. Jordan, R. Scholz, P. Wust, H. Fähling and Roland Felix, J. Magn. Magn. Mater., 1999, 201, 413-419.

5 K. Maier-Hauff, F. Ulrich, D. Nestler, H. Niehoff, P. Wust, B. Thiesen, H. Orawa, V. Budach and A. Jordan, J. Neurooncol., 2011, 103, 317-324. S. Luo, L. F. Wang, W. J. Ding, H. Wang, J. M. Zhou, H. K. Jin, S. F. Su and Ouynagm W.W., OA Cancer, 2014, 2, 2.

7 M. Mahmoudi, H. Hofmann, B. Rothen-Rutishauser and A. Petri-Fink, Chem. Rev., 2012, 112, 2323-2338. Biological, physical, and clinical aspects of hyperthermia:, eds. B. Paliwal, F. W. Hetzel and M. W. Dewhirst, American
Association of Physicists in Medicine by the American Institute of Physics, 1988, pp. 82-110.
9

10

\section{1}

12 W. J. Atkinson, I. A. Brezovich and D. P. Chakraborty, IEEE Trans. Biomed. Eng., 1984, BME-31, 70-75.

R. Hergt, S. Dutz, R. Müller and M. Zeisberger, J. Phys. Condens. Matter, 2006, 18, S2919-S2934.

M. Johannsen, U. Gneveckow, L. Eckelt, A. Feussner, N. WaldÖFner, R. Scholz, S. Deger, P. Wust, S. A. Loening and A. Jordan, Int. J. Hyperth., 2005, 21, 637-647.

U. Gneveckow, A. Jordan, R. Scholz, V. Brüß, N. Waldöfner, J. Ricke, A. Feussner, B. Hildebrandt, B. Rau and P. Wust, Med. Phys., 2004, 31, 1444-1451.

L. Gu, R. H. Fang, M. J. Sailor and J.-H. Park, ACS Nano, 2012, 6, 4947-4954.

M. Marciello, V. Connord, S. Veintemillas-Verdaguer, M. A. Vergés, J. Carrey, M. Respaud, C. J. Serna and M. P. Morales, J. Mater. Chem. B, 2013, 1, 5995.

P. Guardia, R. Di Corato, L. Lartigue, C. Wilhelm, A. Espinosa, M. Garcia-Hernandez, F. Gazeau, L. Manna and T. Pellegrino, ACS Nano, 2012, 6, 3080-3091.

C. Martinez-Boubeta, K. Simeonidis, A. Makridis, M. Angelakeris, O. Iglesias, P. Guardia, A. Cabot, L. Yedra, S. Estradé, F. Peiró, Z. Saghi, P. A. Midgley, I. Conde-Leborán, D. Serantes and D. Baldomir, Sci. Rep., 2013, 3, 1652.

L. Lartigue, P. Hugounenq, D. Alloyeau, S. P. Clarke, M. Lévy, J.-C. Bacri, R. Bazzi, D. F. Brougham, C. Wilhelm and F. Gazeau, ACS Nano, 2012, 6, 10935-10949.

P. Hugounenq, M. Levy, D. Alloyeau, L. Lartigue, E. Dubois, V. Cabuil, C. Ricolleau, S. Roux, C. Wilhelm, F. Gazeau and R. Bazzi, J. Phys. Chem. C, 2012, 116, 15702-15712. P. M. Zélis, G. A. Pasquevich, S. J. Stewart, M. B. Fernández van Raap, J. Aphesteguy, I. J. Bruvera, C. Laborde, B. Pianciola, S. Jacobo and F. H. Sánchez, J. Phys. D. Appl. Phys., 2013, 46, 125006.

J.-P. Fortin, F. Gazeau and C. Wilhelm, Eur. Biophys. J., 2008, 37, 223-228.

A. Makridis, K. Topouridou, M. Tziomaki, D. Sakellari, K. Simeonidis, M. Angelakeris, M. P. Yavropoulou, J. G. Yovos and O. Kalogirou, J. Mater. Chem. B, 2014, 2, 8390-8398. J.-H. Lee, J. Jang, J. Choi, S. H. Moon, S. Noh, J. Kim, J.-G. Kim, I.-S. Kim, K. I. Park and J. Cheon, Nat. Nanotechnol., 2011, 6, 418-422.

M. Creixell, A. C. Bohórquez, M. Torres-Lugo and C. Rinaldi, ACS Nano, 2011, 5, 7124-7129.

M. E. de Sousa, A. Carrea, P. Mendoza Zélis, D. Muraca, O. 
Mykhaylyk, Y. E. Sosa, R. G. Goya, F. H. Sánchez, R. A. Dewey and M. B. Fernández van Raap, J. Phys. Chem. C, 2016, 120, 7339-7348.

C. Munoz-Menendez, I. Conde-Leboran, D. Serantes, R. Chantrell, O. Chubykalo-Fesenko and D. Baldomir, Soft Matter, 2016, 12, 8815-8818.

M. Domenech, I. Marrero-Berrios, M. Torres-Lugo and C. Rinaldi, ACS Nano, 2013, 7, 5091-5101. A. Villanueva, P. de la Presa, J. M. Alonso, T. Rueda, A. Martínez, P. Crespo, M. P. Morales, M. A. GonzalezFernandez, J. Valdés and G. Rivero, J. Phys. Chem. C, 2010, 114, 1976-1981.

I. Marcos-Campos, L. Asín, T. E. Torres, C. Marquina, A. Tres, M. R. Ibarra and G. F. Goya, Nanotechnology, 2011, 22, 205101. Hogan, Nanoscale, 2016, 8, 16053-16064.

M. Levy, C. Wilhelm, N. Luciani, V. Deveaux, F. Gendron, A. Luciani, M. Devaud and F. Gazeau, Nanoscale, 2011, 3, 4402. B. Kozissnik, A. C. Bohorquez, J. Dobson and C. Rinaldi, Int. J. Hyperth., 2013, 29, 706-714.

I. Hilger and W. A. Kaiser, Nanomedicine, 2012, 7, 1443-1459. I. Hilger, Int. J. Hyperth., 2013, 29, 828-834.

K. Mahmoudi, A. Bouras, D. Bozec, R. Ivkov and C.

Hadjipanayis, Int. J. Hyperth., 2018, 1-13.

S. Dutz, M. Kettering, I. Hilger, R. Müller and M. Zeisberger, Nanotechnology, 2011, 22, 265102.

J. Ge, Y. Hu, M. Biasini, W. P. Beyermann and Y. Yin, Angew. Chemie Int. Ed., 2007, 46, 4342-4345.

J. Liu, Z. Sun, Y. Deng, Y. Zou, C. Li, X. Guo, L. Xiong, Y. Gao, F. Li and D. Zhao, Angew. Chemie Int. Ed., 2009, 48, 5875-5879. L. Luo Zheng, V. Vanchinathan, R. Dalal, J. Noolandi, D. J. Waters, L. Hartmann, J. R. Cochran, C. W. Frank, C. Q. Yu and C. N. Ta, J. Biomed. Mater. Res. Part A, 2015, 103, 31573165.

C. Cheng, F. Xu and H. Gu, New J. Chem., 2011, 35, 10721079.

M. B. Fernández van Raap, D. F. Coral, S. Yu, G. A. Muñoz, F. H. Sánchez and A. Roig, Phys. Chem. Chem. Phys., 2017, 19, 7176-7187.

D. F. Coral, P. Mendoza Zélis, M. Marciello, M. del P. Morales, A. Craievich, F. H. Sánchez and M. B. Fernández van Raap, Langmuir, 2016, 32, 1201-1213.

R. C. O'Handley, Modern magnetic materials : principles and applications, Wiley, 2000.

M. E. de Sousa, M. B. Fernández van Raap, P. C. Rivas, P. Mendoza Zélis, P. Girardin, G. A. Pasquevich, J. L.

Alessandrini, D. Muraca and F. H. Sánchez, J. Phys. Chem. C, 2013, 117, 5436-5445.

E. C. Stoner and E. P. Wohlfarth, Philos. Trans. R. Soc. London. Ser. A, Math. Phys. Sci., 1948, 240, 599-642.

M. J. Benitez, O. Petracic, E. L. Salabas, F. Radu, H. Tüysüz, F. Schüth and H. Zabel, Phys. Rev. Lett., 2008, 101, 97206.
D. A. Wiesner and G. Dawson, Glycoconj. J., 1996, 13, 327-33. A. Hanini, L. Lartigue, J. Gavard, A. Schmitt, K. Kacem, C. Wilhelm, F. Gazeau, F. Chau and S. Ammar, RSC Adv., 2016, 6, 15415-15423.

E. Garaio, J. M. Collantes, F. Plazaola, J. A. Garcia and I. Castellanos-Rubio, Meas. Sci. Technol., 2014, 25, 115702. M. E. Cano, A. Barrera, J. C. Estrada, A. Hernandez and T. Cordova, Rev. Sci. Instrum., 2011, 82, 114904.

M. Beković, M. Trlep, M. Jesenik, V. Goričan and A. Hamler, J. Magn. Magn. Mater., 2013, 331, 264-268.

S. Dürr, W. Schmidt, C. Janko, H. P. Kraemer, P. Tripal, F. Eiermann, R. Tietze, S. Lyer and C. Alexiou, Biomed. Eng. / Biomed. Tech., 2013, 58 (S1), 1.

L.-M. Lacroix, J. Carrey and M. Respaud, Rev. Sci. Instrum., 2008, 79, 93909.

V. Connord, B. Mehdaoui, R. P. Tan, J. Carrey and M. Respaud, Rev. Sci. Instrum., 2014, 85, 93904. C. C. Tai and C. C. Chen, in PIERS 2008 Hangzhou, 2008, pp. 610-614.

S. A. Gonzalez, E. M. Spinelli, A. L. Veiga, D. F. Coral, M. B. F. van Raap, P. M. Zelis, G. A. Pasquevich and F. H. Sanchez, in 2017 IEEE 8th Latin American Symposium on Circuits \& Systems (LASCAS), IEEE, 2017, pp. 1-4. S. Stolik, J. . Delgado, A. Pérez and L. Anasagasti, J. Photochem. Photobiol. B Biol., 2000, 57, 90-93.

M. Suzuki, M. Shinkai, H. Honda and T. Kobayashi, Melanoma Res., 2003, 13, 129-135.

M. N. Rylander, Y. Feng, K. Zimmermann and K. R. Diller, Int. J. Hyperth., 2010, 26, 748-764.

D. Cabrera, A. Lak, T. Yoshida, M. E. Materia, D. Ortega, F. Ludwig, P. Guardia, A. Sathya, T. Pellegrino and F. J. Teran, Nanoscale, 2017, 9, 5094-5101.

S. Kobayashi, A. Ohki, M. Tanoue, Y. Inaoka and K. Murase, Open J. Appl. Sci., 2017, 7, 647-660.

J. García-Otero, M. Porto, J. Rivas and A. Bunde, Phys. Rev. Lett., 2000, 84, 167-170.

D. Serantes and D. Baldomir, Open Surf. Sci. J., 2012, 4, 7184.

U. Nowak, R. W. Chantrell and E. C. Kennedy, Phys. Rev. Lett., 2000, 84, 163-166.

M. E. J. Newman and G. T. Barkema, Monte Carlo Methods in Statistical Physics, Oxford University Press, Oxford, 1999. F. Mazuel, A. Espinosa, N. Luciani, M. Reffay, R. Le Borgne, L. Motte, K. Desboeufs, A. Michel, T. Pellegrino, Y. Lalatonne and C. Wilhelm, ACS Nano, 2016, 10, 7627-7638. 


\section{Highlight:}

Integrated approach for the translation of magnetic hyperthermia to clinical uses. Nanoclusters of crystallographically aligned particles, validation in ex vivo melanoma tumour tissue and portable field inductor device. 




$203 \times 150 \mathrm{~mm}(150 \times 150$ DPI $)$ 